\title{
Nerylneryl diphosphate is the precursor of serrulatane, viscidane and cembrane-type diterpenoids in Eremophila species
}

\author{
Oliver Gericke ${ }^{1,2}$, Nikolaj Lervad Hansen 1,2, Gustav Blichfeldt Pedersen 1,2, Louise Kjaerulff ${ }^{3}$, Dan Luo ${ }^{1,2}$, Dan Staerk ${ }^{3}$, \\ Birger Lindberg Møller ${ }^{1,2}$, Irini Pateraki ${ }^{1,2}$ and Allison Maree Heskes ${ }^{1,2^{*}}$ (i)
}

\begin{abstract}
Background: Eremophila R.Br. (Scrophulariaceae) is a diverse genus of plants with species distributed across semi-arid and arid Australia. It is an ecologically important genus that also holds cultural significance for many Indigenous Australians who traditionally use several species as sources of medicines. Structurally unusual diterpenoids, particularly serrulatane and viscidane-types, feature prominently in the chemical profile of many species and recent studies indicate that these compounds are responsible for much of the reported bioactivity. We have investigated the biosynthesis of diterpenoids in three species: Eremophila lucida, Eremophila drummondii and Eremophila denticulata subsp. trisulcata.
\end{abstract}

Results: In all studied species diterpenoids were localised to the leaf surface and associated with the occurrence of glandular trichomes. Trichome-enriched transcriptome databases were generated and mined for candidate terpene synthases (TPS). Four TPSs with diterpene biosynthesis activity were identified: EITPS31 and EITPS3 from E. lucida were found to produce $(3 Z, 7 Z, 11 Z)$-cembratrien-15-ol and 5-hydroxyviscidane, respectively, and EdTPS22 and EdtTPS4, from E. drummondii and E. denticulata subsp. trisulcata, respectively, were found to produce 8,9-dihydroserrulat-14-ene which readily aromatized to serrulat-14-ene. In all cases, the identified TPSs used the cisoid substrate, nerylneryl diphosphate (NNPP), to form the observed products. Subsequently, cis-prenyl transferases (CPTs) capable of making NNPP were identified in each species.

Conclusions: We have elucidated two biosynthetic steps towards three of the major diterpene backbones found in this genus. Serrulatane and viscidane-type diterpenoids are promising candidates for new drug leads. The identification of an enzymatic route to their synthesis opens up the possibility of biotechnological production, making accessible a ready source of scaffolds for further modification and bioactivity testing.

Keywords: Bioactive diterpenoids, Eremophila, cis-prenyltransferase, Terpene synthase, Serrulatanes, Viscidanes, Plant natural products

\section{Background}

Eremophila R.Br. (Scrophulariaceae) is a large and diverse genus of plants endemic to mainland Australia. Members of this genus occur across the continent with

\footnotetext{
* Correspondence: amh@plen.ku.dk

${ }^{1}$ Plant Biochemistry Laboratory, Department of Plant and Environmental Sciences, University of Copenhagen, Thorvaldsensvej 40, DK-1871 Frederiksberg C, Denmark

${ }^{2}$ Center for Synthetic Biology "bioSYNergy", Department of Plant and Environmental Sciences, University of Copenhagen, Thorvaldsensvej 40, DK-1871 Frederiksberg C, Denmark

Full list of author information is available at the end of the article
}

the greatest species diversity found in Western Australia [1]. Species range in form from prostrate ground covers to large shrubs and are found mainly in semi-arid to arid regions. Eremophila is an important source of traditional herbal medicines for many Indigenous Australians [2-5]. Although the species and methods for remedy preparation can differ between communities, leaves are the most frequently used plant part. They are used fresh or dried, boiled, pounded into pastes or mixed with oils to make therapeutic preparations used for treating a wide range of illnesses. Reported uses include treatments for

(c) The Author(s). 2020 Open Access This article is distributed under the terms of the Creative Commons Attribution 4.0 International License (http://creativecommons.org/licenses/by/4.0/), which permits unrestricted use, distribution, and reproduction in any medium, provided you give appropriate credit to the original author(s) and the source, provide a link to the Creative Commons license, and indicate if changes were made. The Creative Commons Public Domain Dedication waiver (http://creativecommons.org/publicdomain/zero/1.0/) applies to the data made available in this article, unless otherwise stated. 
skin and eye infections [2-4], fevers [3], pain [2-4], coughs and colds $[2,3,5]$ gastrointestinal complaints $[2$, 3], and inflammation [3]. Investigations of the specific activity of selected Eremophila spp. extracts have found a range of different bioactivities including anti-viral [6], antibacterial [7-9], anti-cancer [10], and inhibition of ion channels [11]. Diterpenoids, particularly serrulatanes, have been identified as major sources of the observed bioactivity of many of the extracts and have been shown to possess antimalarial [12], antibacterial [13-17], antidiabetic [18, 19] and anti-inflammatory [13] activities. Further reports on the bioactivity of structurally related diterpenoids isolated from Leucophyllum frutescens (also Scrophulariaceae) [20] and several marine gorgonian coral species [21] support this group of molecules as a potential source of new drug leads.

The diterpenoid chemistry of Eremophila is diverse with over 100 different structures reported to-date [12, 15, 17-19, 22]. Linear, macrocyclic, and polycyclic structures are represented across the genus, but no labdane-related diterpenoids (which are often the predominant class found in plants [23]) have been reported. Instead, many of the diterpenoids appear to be C20 analogues of sesquiterpenes with an uncyclized fourth prenyl unit. Because of their unusual structures and potential as drug leads, we set out to identify the enzymes involved in Eremophila diterpenoid biosynthesis.

Terpenes are biosynthesised from linear prenyl diphosphates of different lengths by enzymes belonging to the terpene synthase (TPS) family [24], which are classified into subfamilies based on phylogenetic relationships (TPS-a to TPS-h) [24-26]. The main pathway to diterpenoids in angiosperms involves the sequential activity of two TPSs (class II followed by class I) and leads to the formation of the labdane-related diterpenoids (characterised by a decalin core) [23]. Diterpenes can also be biosynthesised directly from geranylgeranyl diphosphate (GGPP) or nerylneryl diphosphate (NNPP, the all cis isomer of GGPP) by class I TPSs alone to generate linear [27, 28], macrocyclic [29-31] and (poly) cyclic [32-35] products. These enzymes catalyse metal ion dependant ionization of the diphosphate bond of their prenyl diphosphate substrates to generate a reactive carbocation molecule. This intermediate then undergoes a series of rearrangements (e.g. hydride and alkyl shifts, proton transfers, deprotonation and reprotonation) and/or cyclizations [36]. The specific pathways followed in these reaction cascades are dependent on the interactions of the substrate with active site residues and sometimes water molecules. Terpene products are then formed by either proton abstraction from the carbocation to yield diterpene olefins or by water quenching of the carbocation to yield hydroxylated diterpenoids.
The linear prenyl diphosphate precursors used by TPSs are derived from two isomeric C5 building blocks, isopentenyl diphosphate (IPP) and dimethylallyl diphosphate (DMAPP). In head to tail condensation reactions IPP is sequentially joined to DMAPP or an existing longer chain prenyl diphosphate acceptor to build prenyl diphosphates of different chain lengths. Trans-Prenyl diphosphates are the typical substrates of plant TPSs and are biosynthesised by different short-chain transprenyl transferases (trans-PTs) which generate products of specific chain lengths: geranyl diphosphate synthase (GPPS, C10), (E,E)-farnesyl diphosphate synthase $((E, E)$ FPPS, C15), geranylgeranyl diphosphate synthase (GGPPS, C20) and the recently identified geranylfarnesyl diphosphate synthase (GFPPS, C25) [37, 38]. A limited number of plant TPSs are known to use cis-prenyl diphosphates as substrates [32, 34, 39-44]. cis-Prenyl diphosphates are biosynthesised by a family of enzymes known as cis-prenyl transferases (CPTs) which are evolutionarily unrelated to trans-PTs. They also biosynthesize prenyl diphosphates of different chain lengths, which are roughly defined as short (C10-C20), medium (C35-C55), long chain (C80-C95) and very-long-chain (> C50000) [45], the latter being involved in natural rubber biosynthesis [46-48]. Most reported CPTs produce medium and long-chain products like dolichols that are involved in core metabolic processes $[45,49]$. A few studies report CPTs with shortchain biosynthesising activity involved in terpenoid biosynthetic pathways. For example, in Solanum spp. a group of short-chain CPTs has been identified that are involved in mono-, sesqui- and diterpene biosynthesis [32, 39, 40, 44, 50].

Apart from a single report investigating terpene biosynthesis in Eremophila serrulata in which two monoterpene synthases were identified and characterised as multifunctional myrcene/Z-( $\beta$ )-ocimene synthases [51], no studies on the biosynthesis of terpenes in Eremophila are found in the literature. To investigate diterpenoid biosynthesis in Eremophila we selected three species with differing diterpenoid profiles for analysis: E. lucida Chinnock, E. drummondii $\mathrm{F}$. Muell and E. denticulata subsp. trisulcata Chinnock. All species are endemic to southern Western Australia and grow to medium-sized shrubs on a range of soil types in open Eucalyptus woodlands [1]. The ethyl acetate leaf extract of E. lucida is dominated by two diterpenoids: a bicyclic viscidane, 5hydroxyviscida-3,14-dien-20-oic acid (4; Fig. 1) and a macrocyclic compound, 15-hydroxycembra-3,7,11-trien19-oic acid (5; Fig. 1) [18]. Coumpound 4 was identified as an inhibitor of protein-tyrosine phosphatase $1 \mathrm{~B}$ (PTP1B), a potential target for type II diabetes therapeutics [18]. In contrast, the diterpenoid profiles of E. drummondii and $E$. denticulata subsp. trisulcata are characterised by the presence of serrulatanes (Fig. 1) [19, 


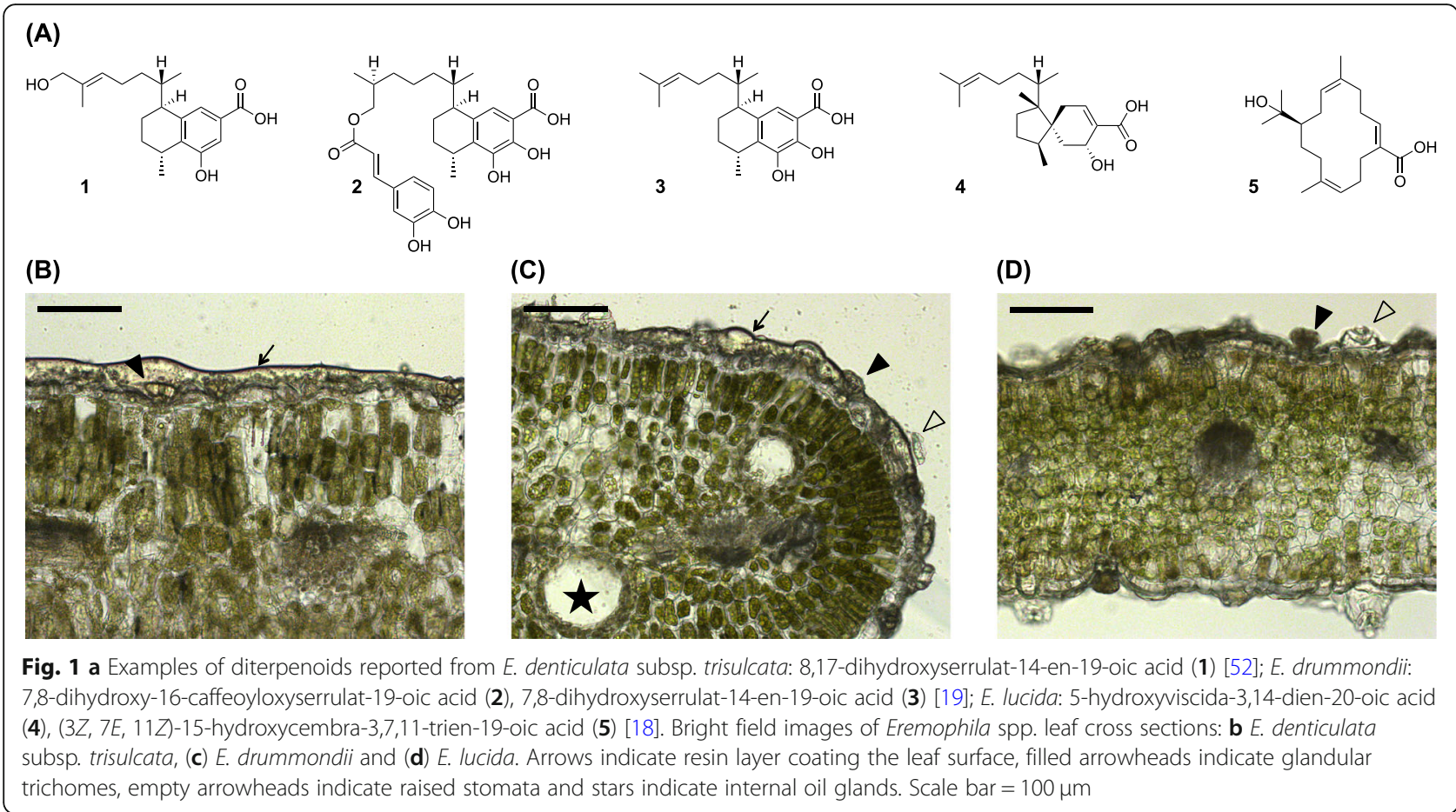

53, 54], several of which from E. drummondii have also been reported to inhibit PTB1B as well as $\alpha$-glucosidase [19].

Here, we describe the identification and functional characterisation of four TPSs that together account for the production of the three major diterpene backbones found across E. lucida, E. drummondii and E. denticulata subsp. trisulcata. In contrast to the majority of known plant diterpene synthases (diTPSs), they use the C20 cisoid precursor, nerylneryl diphosphate (NNPP), as substrate. Accordingly, we also identified one CPT in each species capable of producing NNPP.

\section{Results}

The leaves of $E$. lucida, E. denticulata subsp. trisulcata and E. drummundii are covered by a resinous exudate (Fig. 1). Liquid chromatography-high resolution mass spectrometry

(LC-HRMS) analysis of this resinous layer showed the presence of $m / z$ values expected of diterpenoids reported from these species (Fig. 1 and Additional file 2: Figure S1) $[18,19,54]$. Microscopic examination of leaves revealed the presence of peltate glandular trichomes on both the adaxial and abaxial leaf surfaces of all species. The trichomes were found to consist of a short stalk and a head of eight secretory cells, with combined diameter of $30-40 \mu \mathrm{m}$. Based on our results indicating surfacelocalisation of diterpenoids and in combination with the well-established role of glandular trichomes in terpenoid biosynthesis in numerous species [55], we speculated that they would also have this function in Eremophila.
Consequently, the trichomes were targeted for transcriptomics.

Brushing and ice abrasion methods were not successful at removing the glandular trichomes from the leaf surface, presumably because of their embedded positioning in the epidermis and the large amounts of resinous exudate covering the leaf surface (Fig. 1). Consequently, a novel gland isolation procedure was developed. This procedure involved flash freezing of leaf material sandwiched between two plastic plates, followed by abrupt separation of the two plates. This resulted in resin and glandular trichomes sticking to the surface with minimal adherence of other leaf material. The material adhering to the plates was washed off with pre-chilled RNA isolation/lysis-buffer, the mixture collected, mechanically disrupted and finally extracted for RNA. The resulting RNA was used to generate trichome-enriched transcriptomes using Illumina HiSeq 2500 technology (for transcriptome statistics see Additional file 1: Table S1).

To get an initial indication of the activity of terpenoid biosynthesis in the trichomes, we searched for upstream genes involved in terpenoid metabolism from the mevalonate (MVA) and the 2-C-methyl-D-erythritol 4-phosphate (MEP) pathways using annotated Arabidopsis protein sequences as queries. In the trichome transcriptomes from all three Eremophila species transcripts representing genes from every step in the MVA and MEP pathways were detected (Additional file 1: Table S2).

Transcripts encoding candidate TPSs were identified in the libraries using homology-based searches with 


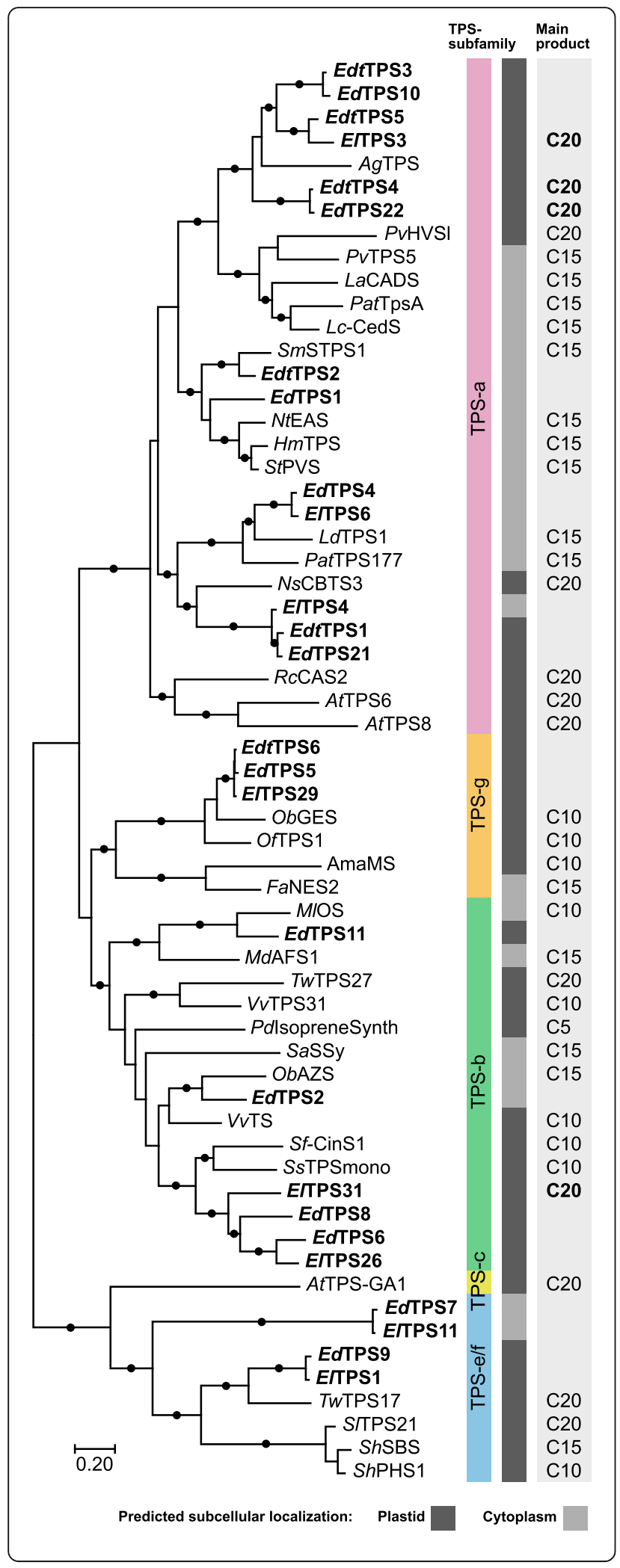

Fig. 2 Phylogenetic analysis of Eremophila TPSs. Maximum likelihood tree of TPSs based on aligned protein sequences calculated using MEGA 7 [56]. Tree is drawn to scale, with branch lengths representing the number of substitutions per site. Filled circles on branches indicate bootstrap support of above $75 \%$ based on 1000 repetitions. Genbank accession numbers are listed in Tables S3 and S4 (Additional file 1).

Subcellular localisation predicted using DeepLoc-1.0 [57]

known TPSs. Of the identified candidates, 6, 12 and 8 likely full length TPSs could be cloned from E. denticulata subsp. trisulcata, E. drummondii and E. lucida trichome cDNA, respectively (Additional file 1: Table S3). Phylogenetic analysis of the candidate TPSs indicated notable expansions in both the TPS-a and TPS-b subfamilies (Fig. 2; Additional file 1: Table S4). Six TPSs were found to form a cluster within the TPS-a clade. The most closely related characterised TPSs to this cluster are Lamiaceae sesquiterpene synthases (sesquiTPSs) making cyclic sesquiterpenes and $P \nu \mathrm{HVS}$, a recently reported diTPS from Prunella vulgaris [35]. Unlike the sesquiTPSs, which were predicted to be localised to the cytosol, all of the Eremophila TPSs of this clade were predicted to be plastid targeted similarly to P $\nu \mathrm{HVS}$. Interestingly, this clade contained many of the most highly expressed TPSs (based on TPM) in the trichomeenriched transcriptome libraries from each species (e.g. ElTPS3, EdTPS22 and EdtTPS4; Additional file 1: Table S3). A second Eremophila specific cluster was observed to group with TPSs involved in the biosynthesis of cyclic monoterpenes in the TPS-b subfamily (Fig. 2). Again, several of these candidates had high TPM values (ElTPS31 and EdTPS6; Additional file 1: Table S3).

All 26 TPS candidates were screened for diTPS activity by Agrobacterium-mediated transient expression in Nicotiana benthamiana [58, 59]. Each TPS was transiently expressed in combinations with either a GGPPS from Coleus forskohlii (CfGGPPS) [60] or an NNPPS from Solanum lycopersicum (SlCPT2) [50]. Gas chromatography-mass spectrometry (GC-MS) analysis of leaf extracts did not show any diterpene products for any of the Eremophila TPSs when coexpressed with CfGGPPS. In contrast, co-expression of either one of ElTPS3, ElTPS31, EdtTPS4 or EdTPS22 with NNPP-forming SICPT2 resulted in diterpene production. ElTPS31 produced $\mathbf{6}$ as a main constituent along with minor amounts of a later eluting product (7), while ElTPS3 produced $\mathbf{8}$ as a main product along with minor products 9 and 10 (Additional file 3: Figure S2). EdtTPS4 and EdTPS22 were found to have identical product profiles appearing as two major constituents, $\mathbf{1 1}$ and 12, along with several minor products (Additional file 4: Figure S3). The candidates with diTPS activity were subsequently cloned into the pet $28 \mathrm{~b}+$ vector, with putative plastid transit sequences removed, for functional testing in $E$. coli. 
Co-expression of ElTPS3, ElTPS31, EdtTPS4 and EdTPS22 with SICPT2 resulted in the same major diterpene products as observed in $N$. benthamiana (Fig. 3). The product profiles of ElTPS3 and EdtTPS4/EdTPS22 when expressed in $E$. coli were, however, somewhat
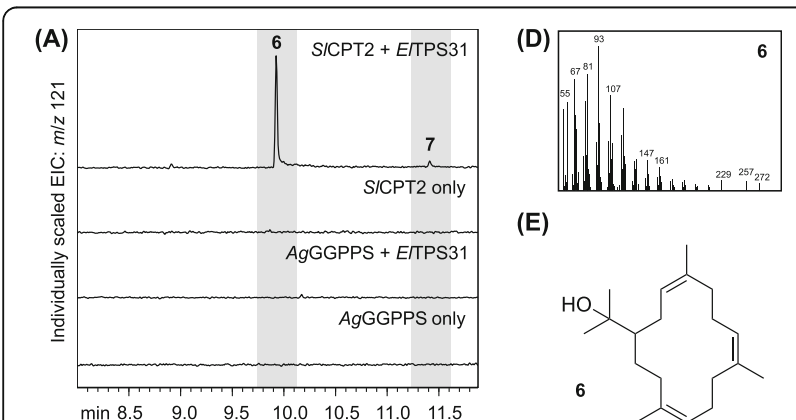

(E)
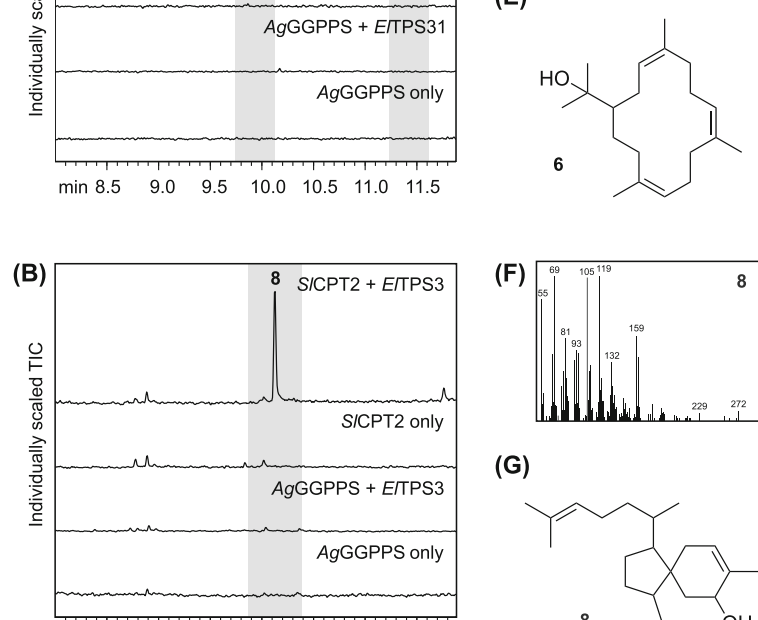

$\begin{array}{lllllll}\min 8.5 & 9.0 & 9.5 & 10.0 & 10.5 & 11.0 & 11.5\end{array}$

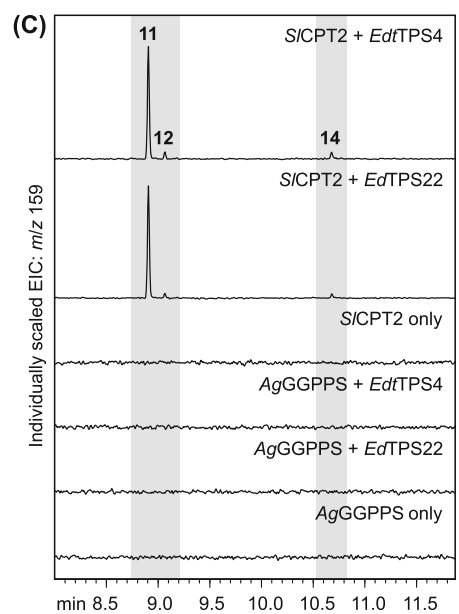

(H)

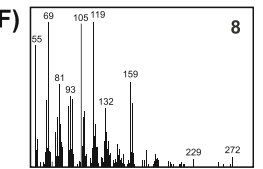

(G)
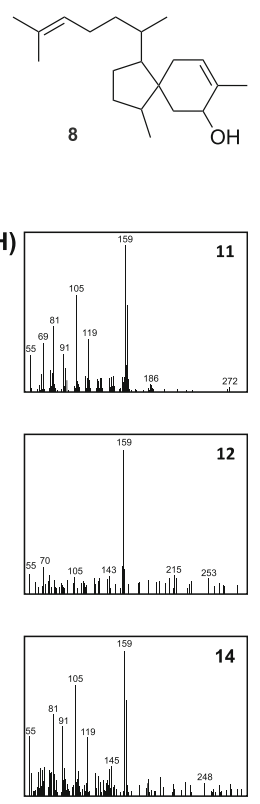

(I)

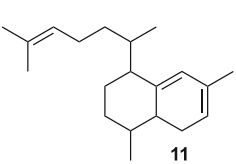

(J)

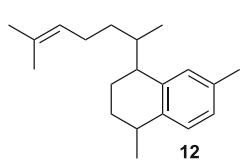

Fig. 3 In vivo functional characterisation of Eremophila TPSs. (A-C) GC-MS chromatograms of hexane extracts of $E$. coli cultures expressing EITPS31, EITPS3, EdtTPS4 and EdTPS22 in combination with either a GGPP synthase (AgGGPPS) or a NNPP synthase (SICPT2). $\mathbf{d}$, $\mathbf{f}$ and $\mathbf{h}$ Mass spectra of major TPS products. $\mathbf{e}, \mathbf{g}$, $\mathbf{i}$ and $\mathbf{j}$ Chemical structures of (3Z,7Z,11Z)-cembratrien-15-ol (6), 5-hydroxyviscidane (8), 8,9-dihydroserrulat-14-ene (11) and serrulat-14-ene (12)

simplified with only trace levels of $\mathbf{9}$ and $\mathbf{1 0}$ detected in strains expressing ElTPS3 and a large peak intensity shift towards 11 in strains expressing EdtTPS4 or EdTPS22.

Each of the major products from ElTPS31, ElTPS3 and $E d t$ TPS4/EdTPS22 was purified from E. coli cultures and their structures elucidated by NMR spectroscopic analysis. The major diterpene products of ElTPS31 and ElTPS3 were identified as $(3 Z, 7 Z, 11 Z)$-cembratrien-15-ol (6; Additional file 1: Table S5) and 5-hydroxyviscidane (8; Additional file 1: Table S6), respectively (Fig. 3). The Z-configuration of each of the double bonds in $\mathbf{6}$ was established by NOE correlations between the olefinic protons and the methyl groups, showing them to be on the same side $(Z=$ zusammen $=$ cis) of the double bond. Similarly, NOE correlations were observed between the two methylene groups attached to the double bonds, further supporting the $Z$ configuration of all double bonds. During the purification of the EdtTPS4/EdTPS22 products, in which a reversephase thin-layer chromatography (RP-TLC) strategy was employed, conversion of $\mathbf{1 1}$ to $\mathbf{1 2}$ was observed by GC-MS analysis. NMR analysis of the resulting sample identified $\mathbf{1 2}$ as serrulat-14-ene (Additional file 1: Table S7). GC-QTOFMS analysis of an extract of $E$. coli cultures expressing EdtTPS4 gave an $[\mathrm{M}+\mathrm{H}]^{+}$ion of $\mathrm{m} / z 273.2569$ (calc. $\mathrm{m} / \mathrm{z}$ 273.2577, 4.4 ppm difference) for peak 11, suggesting a molecular formula of $\mathrm{C}_{20} \mathrm{H}_{32}$ indicating that 12 (with molecular formula of $\mathrm{C}_{20} \mathrm{H}_{30}$ ) likely arises from the aromatization of 11. Development of an alternative purification strategy using solid-phase extraction enabled the isolation of enough $\mathbf{1 1}$ for NMR analysis, which was identified as 8,9-dihydroserrulat-14-ene (Additional file 1: Table S8).

In light of the finding that the major diterpene backbones of these species are derived from NNPP we searched the transcriptome databases for NNPPS candidates belonging to the CPT family. A family of CPTs was identified in all three species with between five and six full-length representatives in each transcriptome (Additional file 1: Table S3). A phylogenetic analysis was carried out to investigate how the Eremophila CPTs are related to characterised angiosperm CPTs (Fig. 4; Additional file 1: Table S9). Overall, the phylogenetic analysis showed the CPTs split in two main clusters: those localised in the plastids and those in the endoplasmic reticulum (ER). Each of these groups was further divided into two sub-clusters. For the ER localised candidates, one cluster was positioned within a clade containing CPTs involved in rubber biosynthesis $[46,48,61]$ and the second cluster was associated with a clade containing long-chain polyprenyl diphosphate synthases that participate in dolichol biosynthesis [48, 62]. Of those Eremophila CPTs predicted to have plastid transit peptides, one group segregated with CPTs of medium and long-chain synthesising enzymes involved in plastid localised polyprenol biosynthesis $[49,63]$ while the other 

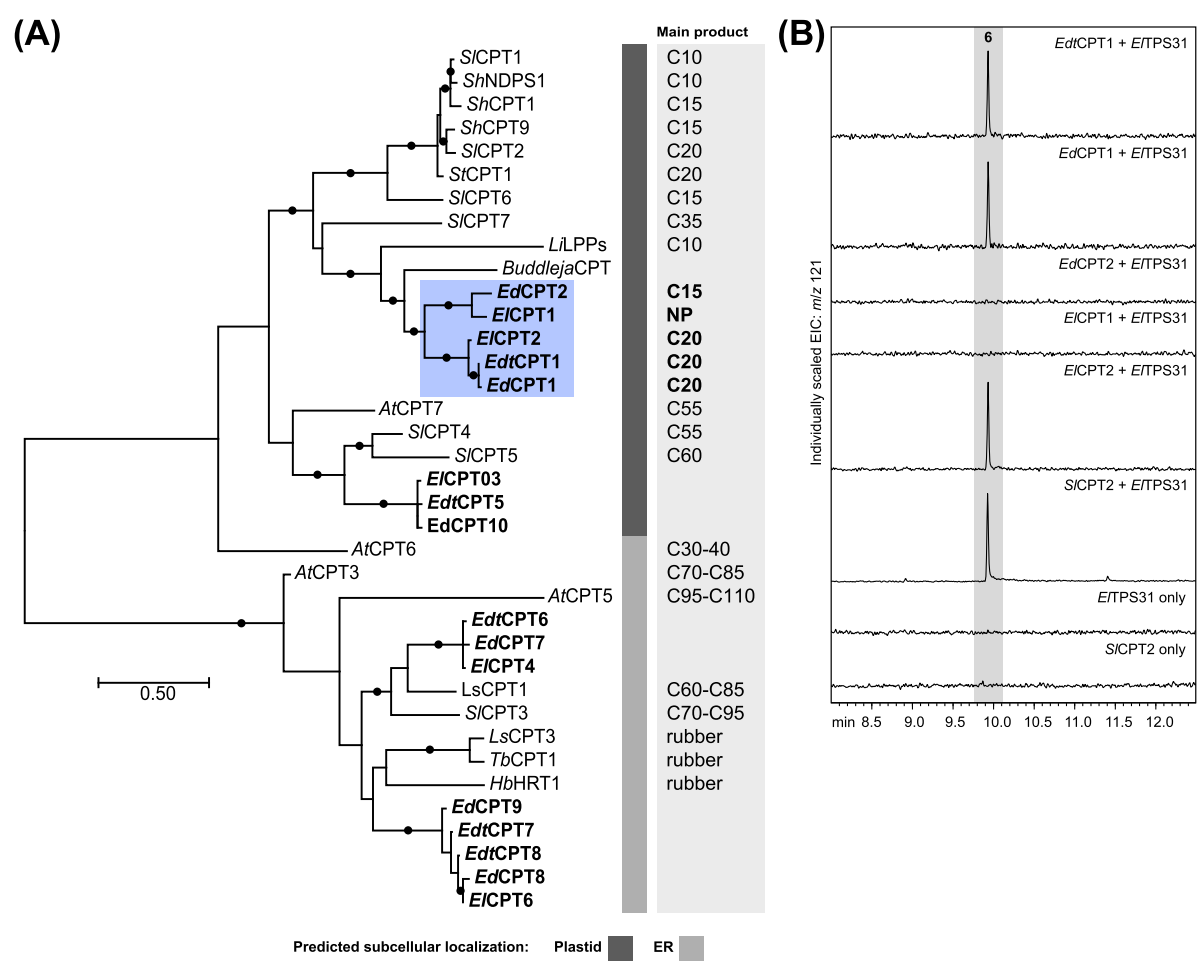

Fig. 4 a Phylogenetic analysis of Eremophila CPTs. Maximum likelihood tree of CPTs based on aligned protein sequences calculated using MEGA 7 [56]. Tree is drawn to scale, with branch lengths representing the number of substitutions per site. Filled circles on branches indicate bootstrap support above 75\% based on 1000 repetitions. Genbank accession numbers are listed in Tables S3 and S9 (Additional file 1). Eremophila CPTs in blue box were functionally characterised. NP = no main product detected. Subcellular localisation predicted using DeepLoc-1.0 [57]. b In vivo functional characterisation of Eremophila CPTs. GC-MS chromatograms of hexane extracts of E. coli cultures expressing Eremophila CPTs in combination with EITPS31

group clustered with short-chain synthesising enzymes that are involved in mono-, sesqui- and diterpene biosynthesis in Solanum spp. and Lavandula $\times$ intermedia [32, 39, 40, 44, 64]. Based on the phylogenetic ties with characterised short-chain CPTs, and the predicted plastid localisation along with high TPM values for three of the candidates (EdCPT1, EdtCPT1 and ElCPT2; Additional file 1: Table S3) relative to the other CPT candidates, we hypothesized that candidates in this latter cluster could be involved in diterpene biosynthesis in Eremophila.

All five candidate CPTs in this cluster were cloned from trichome RNA derived cDNA and tested for NNPP synthesising activity in E. coli. Cell cultures expressing the Eremophila CPTs (truncated to remove putative plastid transit sequences) were lysed and treated with alkaline phosphatase to hydrolyse the diphosphate products to their respective alcohols, extracted with hexane and analysed by GC-MS. EdCPT1, EdtCPT1 and ElCPT2 were found to produce NNPP as their main product and $E d$ CPT2 produced $(Z, Z)$-FPP as the main product (Table 1; Additional file 5: Figure S4) while ElCPT1 produced only trace amounts of NNPP. The CPTs were also co-expressed with the newly established NNPP acceptor,
ElTPS31. In support of the above results, EdCPT1, $E d t \mathrm{CPT} 1$ and ElCPT2 could all combine with ElTPS31 to produce 6 (Fig. 4), whereas no products were observed for combinations with EdCPT2 and ElCPT1.

Leaf extracts from all three Eremophila species were analysed by GC-MS and examined for the presence of the TPS products. Apart from a small signal in E. lucida extracts corresponding to 6 (Additional file 6: Figure S5), none of the other TPS products were detected in the plant extracts.

\section{Discussion}

Involvement of trichomes in the biosynthesis of diterpenoids in Eremophila

Eremophila species are a rich source of novel terpenoids, particularly diterpenoids [22]. Early studies have suggested

Table 1 In vivo functional characterisation of Eremophila CPTs. Products were detected by GC-MS as prenyl alcohols after alkaline phosphatase treatment of lysed E. coli cultures

\begin{tabular}{llllll}
\hline & EdCPT1 & EdCPT2 & EdtCPT1 & ElCPT1 & E/CPT2 \\
\hline Main product & NNPP & $(Z, Z)-F P P$ & NNPP & - & NNPP \\
Minor product & $(Z, Z)-F P P$ & NNPP & $(Z, Z)-F P P$ & NNPP & $(Z, Z)-F P P$ \\
\hline
\end{tabular}


that in many Eremophila species diterpenoids are biosynthesised in glandular trichomes from where they are released to form part of the viscid resins that coat the surface of leaves [53, 65-67]. The three Eremophila species investigated in this study are all characterised by the presence of such a resin as well as short peltate glandular trichomes positioned below the layer of resin (Fig. 1). Analysis of leaf surface extracts indicated the resin from each species contained the diterpenoids of interest (Additional file 2: Figure S1). Furthermore, analysis of the trichome-enriched transcriptomes indicated a high level of activity of the MEP pathway based on TPM values (Additional file 1: Table S2), which is generally known to be involved in diterpenoid biosynthesis [68]. Taken together with the identification of the presently reported diTPSs and CPTs, it is likely that the trichomes are the site of diterpenoid biosynthesis in the Eremophila species examined here. This is in agreement with the known role of glandular trichomes in specialised terpenoid biosynthesis in plants [69-71].

Serrulatane, viscidane and cembrane-type diterpenoids in Eremophila spp. are biosynthesised from the noncanonical terpene precursor, NNPP, by class I type terpene synthases

The majority of diterpenes found in plants are of the labdane-type and derived from the transoid precursor, GGPP. They are biosynthesised via a step-wise process involving the sequential action of class II and class I diTPSs typically from the subfamilies TPS-c and TPS-e/f, respectively [72]. In contrast, each of the major diterpene backbones focused on in this study was found to be biosynthesised from the cis-prenyl diphosphate, NNPP, by the action of a single class I TPS. Based on phylogenetic analyses, the 8,9-dihydroserrulat-14-ene synthases (EdtTPS4 and EdTPS22) and 5-hydroxyviscidane synthase (ElTSP3) belong to the TPS-a subfamily, whereas the $(3 Z, 7 Z, 11 Z)$ cembratrien-15-ol synthase (ElTPS31) belongs to the TPSb subfamily (Fig. 2). Although dominated by sesquiTPSs (TPS-a) and monoterpene synthases (monoTPSs; TPS-b), a few diTPSs have been reported from these subfamilies. For example, macrocyclic diTPSs from multiple plant families form a cluster in the TPS-a subfamily and include macrocyclic diTPSs from the Euphorbiaceae [29, 73-75] and a group of root expressed diTPSs from Arabidopsis [30, 33]. Interestingly, the Eremophila diTPSs in this sub-family are not closely related to these diTPSs but appear to be more closely related to sesquiTPSs and the recently identified diTPS from Prunella vulgaris, PvHVS (Fig. 2) [35]. Similarly, ElTPS31 is more closely related to monoTPSs (Fig. 2) rather than the only other two diTPSs known from the TPS-b subfamily: a pair of orthologous miltiradiene synthases from Tripterygium spp., which accept the bicyclic class II TPS product (+)-copalyl diphosphate [76, 77]. The phylogenetic relationships of the Eremophila diTPSs, suggest these enzymes evolved from sesquiTPS and monoTPS progenitors, with re-acquisition of a plastid targeting sequence in the case of the TPS-a subfamily members. Such a scenario has been suggested for PvHVS [35] and other TPSs where there is evidence for recent changes in substrate specificity, often accompanied by gain or loss of a functional plastid targeting sequence (for example: [7880]).

There are few other reports of TPSs that use cis-prenyl diphosphates as their natural substrates in planta [32, 34, 39-44]. Those that are known, including the presently identified NNPP accepting TPSs from Eremophila, also do not cluster together in the phylogenetic tree but are scattered amongst TPSs accepting GGPP, $(E, E)$ FPP or GPP. Thus, it appears the ability of TPSs to accept NNPP and other cis-prenyl diphosphates has evolved independently multiple times in the TPS family. Indeed, in vitro testing of TPSs with cis-prenyl diphosphates has revealed some latent ability to accept these substrates, even when there is no evidence for them being the natural substrates in planta [35, 41, 80,81]. This suggests that a barrier to the more widespread occurrence of cis-prenyl diphosphate derived terpenoids in plants may be the limited occurrence of short-chain CPTs rather than an inherent inability of TPSs to accept these substrates. Analysis of the terpene biosynthetic gene cluster on chromosome 8 in Solanum spp. provides some insight into how cis-prenyl diphosphate-based terpenoid metabolism could evolve through a process of co-evolution of CPTs and TPSs $[32,41,43,50]$. This cluster contains CPTs with specialised functions as NPP, $(Z, Z)$-FPP and NNPP synthases. They are present in the gene cluster along with cis-substrate accepting TPSs with mono-, sesquiand diterpene synthase activity which have evolved from a common TPS-e/f diterpene synthase. It is conceivable that the appearance of a CPT with shortchain synthesising activity could combine with TPSs with at least partial activity towards the new substrate to provide the initial genetic starting material for the subsequent gene duplications and specialisation of both CPTs and TPSs as observed in Solanum. This process would be facilitated by the commonly observed substrate promiscuity of TPSs (as reviewed by: [82]; see also [60, 81, 83, 84]), and by the ease with which TPSs can gain new functionalities with few amino acid changes (for example: [85-87]).

\section{Proposed reaction pathways catalysed by EITPS3 and EdTPS22/EdtTPS4}

The viscidane and serrulatane backbones are diterpene analogues of acoradiene and cadalane type sesquiterpenes, respectively. Reaction pathways leading to these sesquiterpene backbones are based on studies of TPSs 
which use $(E, E)$-FPP as their natural substrate but they may still be informative for proposing the reaction pathways of ElTPS3 and EdTPS22/EdtTPS4. The acoradiene backbone is formed from $(E, E)$-FPP via the bisabolyl cation which results from a 1,6-ring closure following an initial trans-cis isomerization of the $\mathrm{C} 2-\mathrm{C} 3$ bond of the $(E, E)$-farnesyl cation to the $(Z, E)$-farnesyl cation via the neutral intermediate nerolidyl diphosphate $[88,89]$. After a 1,2-hydride shift (from position $\mathrm{C} 6$ to $\mathrm{C} 7$ ), the resulting homobisabolyl cation is transformed into the acorenyl cation in a 6,10-ring closure. Subsequent proton elimination from the isopropyl tail and formation of the $\mathrm{C} 11-\mathrm{C} 12$ double bond terminates the reaction. The same mechanism may be used to explain the initial steps in the biosynthesis of 5-hydroxyviscidane catalysed by ElTPS3, but without the need of the trans-cis isomerization due to the cis-configuration of the three stereogenic double bonds of the substrate nerylneryl diphosphate. Thus, after the 1,6-ring closure and a 1, 2-hydride shift from C6 to C7 (Additional file 7: Intermediate IIa in Figure S6), a 6,10-ring closure would afford the viscidanyl cation. The reaction is proposed to be terminated by water quenching of the carbocation at $\mathrm{C} 5$ after a 1,5-hydride shift from $\mathrm{C} 5$ to C11 (Additional file 7: Figure S6).

The cadalane type backbones can be derived from two routes that involve 1,10-cyclization [90,91]. In the first route, ionization of $(E, E)$-FPP is followed by direct 1,10 -cyclization to yield a $(E, E)$-germacradienyl cation. In the second route, cyclization is preceded by the previously described trans-cis isomerization pathway resulting in the formation of a $(Z, E)$-germacradienyl cation. Following further rearrangements of the two intermediates the pathways converge at the cadinenyl cation after 1,6-ring closure [90, 91]. Subsequent hydride shifts followed by proton elimination then lead to a range of cadalane type sesquiterpenes [90]. An alternative route follows the same pathway to the bisabolyl cation as reported for acoradiene type sesquiterpene biosynthesis [91]. From the bisabolyl cation further rearrangement and a second ring closure yields the cadinenyl cation. The reaction pathway leading from NNPP to 8,9-dihydroserrulat-14-ene catalysed by EdTPS22/ EdtTPS4 is potentially more likely to follow the latter route given the stereochemistry of the starting substrate (Additional file 7: Figure S6). Thus, without the need of the trans-cis isomerization of the activated nerylneryl cation, 1,6-ring closure to form intermediate I would be followed by a 1,3 hydride shift from C5 to C7 (Additional file 7: Intermediate IIb in Figure S6), which after a 5,10-ring closure would form the serrulatanyl cation. A 1,4 hydride shift from $\mathrm{C} 4$ to $\mathrm{C} 11$ and enzyme catalysed proton abstraction from $\mathrm{C} 5$ would then lead to 8 , 9-dihydroserrulat-14-ene.

\section{Evolution and function of diterpenoids in Eremophila}

A broader examination of the diterpenoids isolated from Eremophila spp. indicates that, based on structural similarities, the pathway described here-a cis-prenyl diphosphate precursor cyclized directly by class I type TPSs-is common to other species in this genus $[15,17,19,22]$. Along with the widespread occurrence of viscidane, serrulatane and cembrane-type diterpenoids, examples from diverse Eremophila spp. exist of linear and bisabolenetype diterpenoids with cis-configured double bonds, which suggests they are also derived from NNPP [92, 93]. Significantly, other genera in Myoporeae and the sister tribe Leucophylleae contain species with serrulatane type diterpenoids $[20,94]$ making it likely that a similar biosynthetic pathway is present in these genera as well. Furthermore, searches for homologous sequences in publicly available transcriptome databases (1KP database [95];) of other Scrophulariaceae species identified a TPS with a putative plastid transit peptide from Anticharis glandulosa (AgTPS, tribe Aptosimeae [96];), that clusters within the subclade of diterpenoid associated TPS-a enzymes from Eremophila (Fig. 2), and a putative short-chain CPT from Buddleja sp. (BuddlejaCPT, tribe Buddlejeae [96];), also predicted to be plastid localised and clustering with Eremophila NNPP producing CPTs (Fig. 4). Taken together, these data suggest that the alternative biosynthetic route to diterpenoids via NNPP may have arisen before the divergence of these separate lineages in Scrophulariaceae.

To date, Eremophila (and potentially related genera as described above) is the only known example of a lineage of plants that has evolved such extensive diterpenoid chemistry derived largely from the alternative cisoid precursor, NNPP. The broadened chemical diversity resulting from the use of this alternative substrate may offer particular selective advantages, which could explain the remarkable abundance and diversity of these unusual diterpenoids across the entire genus. However, the biological functions of the diterpenoids found in Eremophila species remain uncharacterised. The viscid resin of which they are a part is thought to be an adaptation to aridity mediated by its ability to reduce water loss by increasing resistance to transpiration and by lowering leaf temperature by increasing reflectance of sunlight $[65,97]$. It is also likely that the resin and the diterpenoids therein are involved in defence against herbivores and pathogens. Although again there is no data available relating to this aspect of Eremophila biology, in vitro studies on the bioactivity of serrulatanes show a broad range of antimicrobial activities [12-14, 16, 17, 20]. Adding credence to the idea that serrulatanes may have an antimicrobial function in planta, bees have been reported to collect serrulatane-containing resin from the leaves of the closely related species, Myoporum 
insulare, for making bee-glue, an antiseptic material used by bees to seal their hives [94].

\section{Conclusions}

Our study has identified a biosynthetic route to three of the major diterpene backbones found in Eremophila species. The identified CPTs and TPSs are the starting points of biosynthetic networks involving multiple enzyme-catalysed steps which lead to the more complex and bioactive diterpenoids characteristic of species in this genus, many of which show promise as new drugs or drug leads. The finding that trichomes are the likely site of biosynthesis of diterpenoids and development of trichome-enriched transcriptome databases is providing valuable knowledge and resources that can be used to identify downstream terpenoid biosynthetic enzymes, $[98,99]$.

In this work we show that NNPP is the precursor for all three types of diterpenoids investigated here. The broad distribution of these and similar compounds across Eremophila and related genera suggests this alternative pathway to specialised diterpenoids is common across the plant lineage. With its species richness and broad geographic distribution across Australia, Eremophila thus provides an eminent model system for the study of the evolution of terpenoid chemical diversity.

\section{Materials and methods}

\section{Plant material and glandular trichome RNA isolation}

Plant material was harvested from plants growing in the greenhouse at the University of Copenhagen (Frederiksberg, Denmark) under natural light supplemented with growth lights during winter months $(7 \mathrm{am}-7 \mathrm{pm})$ with an average day/night temperature of $18^{\circ} \mathrm{C}$. Eremophila lucida and E. drummondii material was sourced as described in Tahtah et al. [18] and Wubshet et al. [19], respectively. The E. denticulata subsp. trisulcata specimen was sourced from the State Flora Nursery of South Australia. Voucher specimens of E. lucida (UCPHPLEN-AH4), E. drummondii (UCPH-PLEN-AH3) and $E$. denticulata subsp. trisulcata (UCPH-PLEN-AH6) have been deposited at Herbarium $\mathrm{C}$, National History $\mathrm{Mu}-$ seum, University of Copenhagen.

To isolate glandular trichomes from Eremophila spp. a novel trichome isolation method based on surface contact freezing was developed. Fresh leaves were tightly sandwiched between two plastic plates and frozen on dry ice. The plastic plates with leaves were exposed to room temperature for $10 \mathrm{~s}$ and then abruptly opened leaving trichomes and resin with minimal other leaf material attached to the plates. The plastic plates were washed down with $1.5 \mathrm{~mL}$ pre-chilled lysis-buffer (RNAqueous-Micro Total RNA Isolation Kit, Thermo Fisher Scientific) supplemented with 1:10 Plant RNA Isolation
Aid (Thermo Fisher Scientific) and $300 \mathrm{mg}$ PVP40. The lysis solution was collected into $2 \mathrm{~mL}$ round bottom Eppendorf tubes containing glass beads of different sizes $(1.5 \mathrm{~mm}, 1 \mathrm{~mm}$ and $0.5 \mathrm{~mm}$ diameter) and subjected to a cell disruption step ( 3 cycles of $3 \mathrm{~min}$ at $3000 \mathrm{rpm}$ in a TissueLyser II, QIAGEN, Hilden, Germany), with cooling of samples on dry ice for 2 min between cycles. The lysed trichome samples were centrifuged at $20000 \mathrm{~g}$ for $10 \mathrm{~min}$. The supernatant was transferred to a binding column provided with the RNAqueous-Micro Total RNA Isolation Kit. Total RNA was isolated following standard kit protocol conditions and with on-column DNA digestion with supplied DNase I. RNA integrity and concentration was determined using the RNA-nano assay on the Agilent 2100 Bioanalyzer (Agilent Technologies, Santa Clara, CA, USA).

\section{Transcriptome analysis}

RNA-seq libraries were generated with TruSeq Stranded mRNA LT Sample Prep Kit (Illumina San Diego, USA) using poly-A selection. Library preparation and sequencing was conducted by Macrogen (Seoul, South Korea) with paired ends $(2 \times 101 \mathrm{bp})$ on a HiSeq 2500 (Illumina), according to the manufacturer's instructions. Transcriptome assembly was carried out by Sequentia Biotech SL. A quality check was performed on the raw sequencing data using BBDuk (https://sourceforge.net/ projects/bbmap/), where minimum read length was set to $35 \mathrm{bp}$ and the Phred quality score to 35 . The high quality reads were used as input to perform transcriptome assembly after normalization (with Trinity v2.1.1) [100]. Quality control and filtering of the transcriptome assembly was conducted in three steps. First, the expression levels of all the transcripts were quantified with the software Kallisto [101] and then all transcripts with no expression levels were removed. Second, to reduce the redundancy of the dataset, for each gene only the isoform encoding the longest protein was retained; for the non-coding transcripts, the longest sequence was kept. Finally, all the transcripts having a match with a nonplant organism were filtered out. To obtain the expression quantification of the assembled transcripts in the three samples, the trimmed reads were processed with the software Kallisto and TPM (Transcripts Per Million) values were calculated for all the transcripts. A summary of the transcriptomic data is given in (Additional file 1: Table S2). In addition, de novo transcriptomes were generated with the RNAseq assembly tool of the CLC genomic assembly software (version 11, QIAGEN) using default settings.

The putative function of assembled transcripts as encoding CPTs or TPSs was inferred using two approaches. First, a BLAST based homolog search was applied on the generated transcriptome libraries using 
massblast (https://github.com/averissimo/mass-blast). Secondly, transcripts were scanned for PFAM domains with HMMER (version 3.1b1), using HMM models for the N-terminal (Acc PF01397.20) and C-terminal (Acc PF03936.15) part of terpene synthases (Pfam-A v29). Eventually, the open reading frame-prediction tool of the CLC Main Workbench (version 8.0.1, QIAGEN) was used to identify coding sequences. Phylogenetic analyses were carried out using MEGA 7 [56] as described in Heskes et al. [102].

For the MVA and MEP pathway analysis annotated Arabidopsis thaliana genes of the MEP and MVA pathways were selected from the NCBI Protein database and used to query the Eremophila trichome transcriptomes using tBLASTn with an E-value cutoff of 1E-10. All BLAST hits were checked for open reading frames of at least 200 amino acids and translated into protein sequences. BLAST hits with a minimum overall identity and query coverage of $50 \%$ were selected.

\section{Microscopic analysis of leaf cross sections}

Fresh leaf material was embedded in 5\% agarose and sectioned in $20 \mu \mathrm{m}$ sections using a HM $650 \mathrm{~V}$ vibrating microtome (Microm International, Walldorf, Germany). Sections were mounted in water for imaging using a DMI 4000B inverted microscope (Leica Microsystems, Wetzler, Germany). True color imaging was done under bright field at $20 \times$ magnification. Microscopic images were taken by Leica software and further processed using ImageJ (version 1.51j8).

\section{Functional characterisation of biosynthetic candidate genes in $\mathrm{N}$. benthamiana}

To characterise the function of putative CPTs and TPSs from Eremophila, cloned candidates were tested using Agrobacterium-mediated transient expression in Nicotiana benthamiana. Generation of cDNA from isolated leaf RNA for each Eremophila spp. was done using the iScript cDNA Synthesis Kit (Biorad, Hercules, CA). Gene specific primers (Additional file 1: Table S10) were designed with USER overhangs to amplify full length coding sequences of candidates from generated Eremophila cDNA libraries. A DNA fragment of the coding sequence of SlCPT2 [50] was obtained by commercial synthesis (Thermo Fisher Scientific). Where candidates were not predicted to be full length in the transcriptomic databases transcripts were elongated to full length using homologous sequences found within the three generated Eremophila transcriptomes. For transient expression in $N$. benthamiana, amplicons of coding sequences were integrated into the pCAMBIA130035Su vector by USER cloning [103]. Competent Agrobacterium AGL-1 cells were transformed with plasmid DNA and T-DNA encoded target genes were transiently co- expressed in 4-6 week old $N$. benthamiana plants [58, 59] together with the gene silencing suppressor p19 [104] and the gene encoding the C. forskohlii enzyme, 1deoxy-d-xylulose 5-phosphate synthase (CfDXS) [60]. Six days post-infiltration, two leaf discs $(3 \mathrm{~cm}$ diameter) from individual leaves were excised and extracted in 1 $\mathrm{mL}$ hexane at room temperature for $1 \mathrm{~h}$ on an orbital shaker at $220 \mathrm{rpm}$. Plant material was collected by centrifugation and the organic phase was transferred to GC vials for GC-MS analysis.

\section{GC-MS analysis}

Samples were analysed by GC-MS using a Shimadzu GCMS-QP2010 Ultra (Shimadzu, Kyoto, Japan) fitted with an HP-5MS UI column $(20 \mathrm{~m} \times 0.18 \mathrm{~mm}$ i.d., $0.25 \mu \mathrm{m}$ film thickness; Agilent) using $\mathrm{H}_{2}$ as the carrier gas. The injection port was operated in splitless mode with a starting temperature of $40^{\circ} \mathrm{C}$ which was held for $1 \mathrm{~min}$ and then ramped to $250^{\circ} \mathrm{C}$ over $4 \mathrm{~min}$. The GC oven program was as follows: $60^{\circ} \mathrm{C}$ for $1 \mathrm{~min}$, ramp to $150{ }^{\circ} \mathrm{C}$ at $30^{\circ} \mathrm{C} \mathrm{min}^{-1}$, ramp to $250{ }^{\circ} \mathrm{C}$ at $15^{\circ} \mathrm{C} \mathrm{min}^{-1}$, ramp to $290^{\circ} \mathrm{C}$ at $30^{\circ} \mathrm{C} \mathrm{min}{ }^{-1}$, hold for $3 \mathrm{~min}$. The MS used electron impact (EI) ionisation with the ion source voltage and temperature set to $70 \mathrm{eV}$ and $300^{\circ} \mathrm{C}$, respectively. For analysis of $E$. coli strains expressing Eremophila CPTs and treated with phosphatase the oven program was started at $40^{\circ} \mathrm{C}$. Data was analysed using GCMSsolution software v4.20 (Shimadzu).

\section{Characterisation of ETTPS3, ETTPS31, EdtTPS4 and EdTPS22} in E. coli and isolation of $6,8,11$ and 12

Coding sequences of N-terminally truncated Eremophila TPSs (ElTPS3 $\Delta 1-23, E l \mathrm{TPS} 31 \Delta 1-54$, EdtTPS4 $\Delta 1-59$ and $E d T P S 22 \Delta 1-59)$ were cloned into the pet $28 \mathrm{~b}+$ expression vector). Each TPS construct was used to co-transform E. coli EXPRESS BL21 competent cells (Lucigen, Middleton, WI) along with pIRS [105] and pACYCDuet vector carrying either Abies grandis GGPP synthase [106] or N-terminally truncated Solanum lycopersicum NNPP synthase (SlCPT2) [50]. $2 \mathrm{~mL}$ cultures were grown at $37^{\circ} \mathrm{C}$ until $\mathrm{OD}_{600} 0.8$ was reached, cooled to $16^{\circ} \mathrm{C}$, and induced with the addition of IPTG (final concentration $1 \mathrm{mM})$. The cultures were then grown at $18^{\circ} \mathrm{C}$ for $48 \mathrm{~h}$ at $200 \mathrm{rpm}$, centrifuged and $1 \mathrm{~mL}$ aliquots of supernatant were extracted with $0.3 \mathrm{~mL}$ hexane at room temperature for $1 \mathrm{~h}$ on an orbital shaker at $220 \mathrm{rpm}$. The resulting hexane extracts were analysed by GC-MS as for $N$. benthamiana samples. For compound purification, $200 \mathrm{~mL}$ cultures were grown in unbaffled $2 \mathrm{~L}$ conical flasks with the above-described conditions. Largescale cultures were centrifuged and the supernatant extracted with an equal volume of hexane twice. The hexane extracts were reduced in volume by rotary evaporation and the concentrate fractionated using RP-TLC with 
methanol as the mobile phase to give $\mathbf{6 , 8}$ and $\mathbf{1 2}$. To purify 11, the concentrated hexane extract was applied onto a Dual layer florisil/ $\mathrm{Na}_{2} \mathrm{SO}_{4}$ SPE cartridge $(6 \mathrm{~mL}$, Supelco, PA, USA) and eluted with $1 \%$ ethyl acetate in hexane.

To obtain accurate mass data on the TPS products, extracts of E. coli cultures expressing ElTPS3, ElTPS31, EdtTPS4 and EdTPS22 with SlCPT2 were analysed by GC-QTOF-MS using a Scion 456-GC coupled to a MicroTOF II MS equipped with an APCI source (Bruker Daltonik, Bremen, Germany). Samples were injected in splitless mode with an injection port temperature of $250{ }^{\circ} \mathrm{C}$. The GC was fitted with a $30 \mathrm{~m}$ BR- $5 \mathrm{~ms}$ column (5\% phenyl, 95\% dimethyl arylene siloxane; Bruker) with $250 \mu \mathrm{m}$ ID and $0.25 \mu \mathrm{m}$ film thickness. The carrier gas was $\mathrm{H}_{2}$ with a constant linear velocity of $30 \mathrm{~cm} \mathrm{~s}^{-1}$. The oven program was as follows: initial temperature of $60^{\circ} \mathrm{C}$ held for $1 \mathrm{~min}$, followed by a linear ramp to $130^{\circ} \mathrm{C}$ at $20^{\circ} \mathrm{C} \mathrm{min}^{-1}$, which was then ramped to $250^{\circ} \mathrm{C}$ at $4{ }^{\circ} \mathrm{C} \mathrm{min}^{-1}$. Finally the oven was ramped to $290^{\circ} \mathrm{C}$ at $30{ }^{\circ} \mathrm{C} \mathrm{min}^{-1}$ and held for $4 \mathrm{~min}$. The APCI source was operated in positive ionization mode with the following settings: capillary voltage, $3000 \mathrm{~V}$; corona discharge needle, $2000 \mathrm{nA}$; nebulizer gas pressure, 3 bar; dry gas flow, $2.5 \mathrm{~L} \mathrm{~min}^{-1}$; dry gas temperature, $250^{\circ} \mathrm{C}$. A mass range of 50 to $700 \mathrm{~m} / z$ was used.

\section{Characterisation of Eremophila CPTs in E. coli}

To test for the ability of the selected Eremophila CPTs to produce NNPP, the same $E$. coli system as employed for TPS characterisation was used. Coding sequences of Nterminally truncated Eremophila CPTs (EdCPT1 $11-58$, $E d C P T 2 \Delta 1-58, \quad E d t C P T 1 \Delta 1-58, \quad E l C P T 1 \Delta 1-60$ and ElCPT2 $\Delta 1-58)$ were cloned into pACYC-Duet vector and used to co-transform E. coli EXPRESS BL21 competent cells (Lucigen, Middleton, WI) with pIRS [105] and either empty pet $28 \mathrm{~b}+$ or pet28b+:ElTPS31. Culture conditions were the same as for TPS characterisation except that $15 \mathrm{~mL}$ cultures of the strains not expressing ElTPS31 were grown. These cultures were subjected to a cell lysis procedure using a cell disruptor (Constant Systems Ltd., Northants, UK) set to $25 \mathrm{kpsi}$. The lysate was adjusted to $20 \mathrm{~mL}$ with water and centrifuged $8000 \mathrm{~g}$ for $20 \mathrm{~min} .10$ $\mathrm{mL}$ of supernatant was treated with $20 \mu \mathrm{L}$ calf intestinal phosphatase (New England Biolabs, Ipswich, MA) and incubated overnight at $37^{\circ} \mathrm{C}$. Samples were then extracted twice with $1 \mathrm{~mL}$ hexane. Hexane extracts were combined and concentrated to $50 \mathrm{uL}$ under a stream of $\mathrm{N}_{2}$ and analysed by GC-MS. Strains expressing ElTSP31 were prepared and analysed as for TPS characterisation.

\section{Metabolite analysis of Eremophila spp.}

For GC-MS analysis of Eremophila spp. freshly harvested leaves were ground under liquid $\mathrm{N}_{2}$, extracted in hexane while shaking for $1 \mathrm{~h}$ at $24^{\circ} \mathrm{C}$. Hexane samples were analysed by GC-MS as per E. coli and N. benthamiana samples. For LC-HRMS analysis freshly harvested leaves were dipped for $15 \mathrm{~s}$ in $100 \%$ ethyl acetate. The extracts were dried for $1 \mathrm{~h}$ in a speedvac centrifuge and resuspended in $80 \%$ acetonitrile. Acetonitrile extracts were analysed using an Ultimate 3000 UHPLC+ Focused system (Dionex Corporation, Sunnyvale, CA) coupled to a Bruker Compact ESI-QTOF-MS (Bruker) system. Samples were separated on a Kinetex XB-C18 column $(100 \times 2.1 \mathrm{~mm}$ ID, $1.7 \mu \mathrm{m}$ particle size, $100 \AA$ pore size; Phenomenex Inc., Torrance, CA) maintained at $40{ }^{\circ} \mathrm{C}$ with a flow rate of $0.3 \mathrm{~mL} \mathrm{~min}^{-1}$ and mobile phase consisting of $0.05 \%(\mathrm{v} / \mathrm{v})$ formic acid in water (solvent A) and $0.05 \%(\mathrm{v} / \mathrm{v})$ formic acid in acetonitrile (solvent $\mathrm{B}$ ). The LC method was as follows: $0-1 \mathrm{~min}, 10 \% \mathrm{~B} ; 1-23$ min, $10-100 \%$ B; $23-25$ min, $100 \%$; 25-25.5 min, $100-$ 10\%; $25.5-30.5 \mathrm{~min}, 10 \% \mathrm{~B}$. Mass spectra were acquired in positive ion mode with the following ESI settings: capillary voltage, $4000 \mathrm{~V}$; end plate offset, $-500 \mathrm{~V}$; dry gas temperature, $220^{\circ} \mathrm{C}$; dry gas flow of $8 \mathrm{~L} \mathrm{~min}^{-1}$; nebulizer pressure, 2 bar. Data were analysed using DataAnalysis 4.1 (Bruker).

\section{NMR spectroscopy}

Nuclear magnetic resonance spectroscopy (NMR) experiments were recorded in $\mathrm{CDCl}_{3}$ on a $600 \mathrm{MHz}$ Bruker Avance III instrument at a proton frequency of 600.13 $\mathrm{MHz}$, using a $1.7 \mathrm{~mm}$ cryogenically cooled TCI probehead. All NMR experiments were performed in automation (temperature equilibration to $300 \mathrm{~K}$, optimization of lock parameters, gradient shimming, and setting of receiver gain) using IconNMR ver 4.2 (Bruker Biospin, Karlsruhe, Germany). ${ }^{1} \mathrm{H}$ NMR spectra were acquired with $30^{\circ}$-pulses and $64 \mathrm{k}$ data points. Standard 2D homo- and heteronuclear experiments were acquired with 2048 or 1730 (HSQC) data points in the direct dimension and 512 (DQF-COSY) or 256 (multiplicity-edited HSQC and HMBC) data points in the indirect dimension. Topspin ver. 3.5 (Bruker Biospin) was used for acquisition and processing of NMR data.

\section{Supplementary information}

Supplementary information accompanies this paper at https://doi.org/10. 1186/s12870-020-2293-X.

\footnotetext{
Additional file 1: Table S1 Transcriptome sequencing statistics. Table S2. MVA and MEP pathway analysis. Table S3. List of candidate terpene synthases (TPSs) and cis-prenyl transferases (CPTs) studied in this work. E. lucida $=E l$, E. drummondii $=E d$ and $E$. denticulata subsp. trisulcata $=E d t$. DeepLoc-1.0 was used to predict protein subcellular localisation [57]. Table S4. Protein sequences used in the terpene synthase phylogenetic analysis. Table S5. ${ }^{1} \mathrm{H}(600 \mathrm{MHz})$ and ${ }^{13} \mathrm{C}(150 \mathrm{MHz})$ data of $(3 Z, 7 Z, 11 Z)-$ cembratrien-15-ol (6) in $\mathrm{CDCl}_{3}$. Table S6. ${ }^{1} \mathrm{H}(600 \mathrm{MHz})$ and ${ }^{13} \mathrm{C}(150$ $\mathrm{MHz}$ ) data of 5-hydroxyviscidane (8) in $\mathrm{CDCl}_{3}$. Table S7. ${ }^{1} \mathrm{H}(600 \mathrm{MHz})$ and ${ }^{13} \mathrm{C}(150 \mathrm{MHz})$ data of serrulat-14-ene $(\mathbf{1 2})$ in $\mathrm{CDCl}_{3}$. Table S8. ${ }^{1} \mathrm{H}$ $(600 \mathrm{MHz})$ and ${ }^{13} \mathrm{C}(150 \mathrm{MHz})$ data of 8,9-dihydroserrulat-14-ene (11) in
} 
$\mathrm{CDCl}_{3}$. Table S9. Protein sequences used in the cis-prenyl transferase phylogenetic analysis. Table S10. List of primers used in this study.

Additional file 2: Figure S1. Liquid chromatography-high-resolution mass spectrometry (LC-HRMS) extracted ion chromatograms (EIC) of ethyl acetate leaf-surface wash extracts prepared from E. lucida, E. denticulata subsp. trisulcata and E. drummondii. Depicted ElCs represent the combined $\mathrm{m} / \mathrm{z}$ values of the calculated $[\mathrm{M}+\mathrm{H}]^{+}$ions $( \pm 0.05)$ of known diterpenoids reported from these species. E. lucida: 321.2424 [18]; E. denticulata subsp. trisulcata: 333.2066, 335.2221 [52, 54]; E. drummondii; $333.2058,335.2221,363.1809,349.2014,351.2169,513.2474,527.2638$, $421.2586,435.2744,367.2121,409.2226,451.2332,365.1964$ [19, 54]. Predicted molecular formulas are within $\pm 2.0 \mathrm{ppm}$ error of calculated values.

Additional file 3: Figure S2. In planta functional characterisation of ETTPS31 and ETTPS3. (A) and (B) GC-MS chromatograms of hexane extracts of N. benthamiana leaves transiently expressing EITPS31 and EITPS3 in combination with either GGPP synthase (CFGGPPS) or NNPP synthase (SICPT2). (C) Mass spectra of TPS products.

Additional file 4: Figure S3. In planta functional characterisation of EdtTPS4 and EdTPS22. (A) GC-MS chromatograms of hexane extracts of $N$. benthamiana leaves transiently expressing EdtTPS4 and EdTPS22 in combination with either GGPP synthase (CFGGPPS) or NNPP synthase (SICPT2). (B) Mass spectra of main TPS products. (C) Mass spectra of minor products (inset window of $\mathbf{A}$ )

Additional file 5: Figure S4. (A) In vivo functional characterisation of Eremophila CPTs. GC-MS chromatograms of hexane extracts of E. coli cultures treated with alkaline phosphatase. (B) Mass spectra of prenyl alcohols: nerylnerol (a), (2Z,6Z)-farnesol (b) and nerol (c).

Additional file 6: Figure S5. (A) GC-MS analysis of a hexane extract of E. lucida leaves. Only $\mathbf{6}$, the product of EITPS31, was detected in the plant. Comparison trace is of an extract of $N$. benthamiana leaves transiently expressing EITPS31 with NNPPS (SICPT2). (B) Mass spectrum of $\mathbf{6}$ produced by heterologous expression in $N$. benthamiana compared to $E$. lucida leaf extract.

Additional file 7: Figure S6. Proposed reaction pathways catalysed by EdTPS22/EdtTPS4 to 8,9-dihydroserrulat-14-ene and EITPS3 to 5hydroxyviscidane from nerylneryl diphosphate.

\section{Abbreviations}

(E,E)-FPPS: (E,E)-farnesyl diphosphate synthase; CPT: Cis-prenyl transferases; diTPS: diterpene synthase; DMAPP: Dimethylallyl diphosphate; GC-MS: Gas chromatography-mass spectrometry; GFPPS: Geranylfarnesyl diphosphate synthase; GGPP: Geranylgeranyl diphosphate; GGPPS: Geranylgeranyl diphosphate synthase; GPP: Geranyl diphosphate; GPPS: Geranyl diphosphate synthase; IPP: Isopentenyl diphosphate; LC-HRMS: Liquid chromatographyhigh resolution mass spectrometry; MEP: 2-C-methyl-D-erythritol 4phosphate; monoTPSs: monoterpenes synthases; MVA: Mevalonate; NMR: Nuclear magnetic resonance; NNPP: Nerylneryl diphosphate; RPTLC: Reverse-phase thin-layer chromatography; sesquiTPS: sesquiterpene synthase; TPM: Transcripts per million; TPS: Terpene synthase; trans-PTs: transprenyl transferases

\section{Acknowledgements}

The authors thank the Core Metabolomics Platform (Plant Biochemistry Laboratory, University of Copenhagen) for technical support with metabolite analyses and the greenhouse personnel at the University of Copenhagen for growing and caring for the plants.

\section{Authors' contributions}

$\mathrm{AMH}, \mathrm{BLM}$ and IP conceived the original research idea. OG and $\mathrm{AMH}$ performed the majority of experiments, and carried out GC-MS and LC-HRMS analyses. NLH and GBP contributed to the identification and cloning of TPSs and the design and execution of enzyme characterisation experiments. LK, DL and DS performed NMR experiments and structure elucidation of TPS products. $\mathrm{AMH}$ and $\mathrm{OG}$ wrote the article with all other authors contributing. All authors read and approved the final manuscript.

\section{Funding}

This work was financially supported by the Center for Synthetic Biology (University of Copenhagen Excellence Program for Interdisciplinary Research), by a European Research Council Advanced Grant to BLM (ERC-2012-

ADG_20120314), and by a Novo Nordisk Foundation Interdisciplinary Synergy Programme grant (NNF16OC0021616). The NMR system used in this work was acquired through a grant from "Apotekerfonden af 1991", The Carlsberg Foundation, and the Danish Agency for Science, Technology and Innovation via the National Research Infrastructure fund. The funding bodies listed played no role in the design of the study, collection, analysis, and interpretation of data and in writing the manuscript.

\section{Availability of data and materials}

The RNA-seq data generated during the current study have been submitted to the Sequence Read Archive (SRA) at NCBI with the following accession number: PRJNA601673. The cDNA sequences of Eremophila TPSs and CPTs reported in this study are available through GenBank. Accessions numbers are listed in Table S3 (Additional file 1).

\section{Ethics approval and consent to participate}

Not applicable.

\section{Consent for publication}

Not applicable.

\section{Competing interests}

The authors declare that they have no competing interests.

\section{Author details}

${ }^{1}$ Plant Biochemistry Laboratory, Department of Plant and Environmental Sciences, University of Copenhagen, Thorvaldsensvej 40, DK-1871 Frederiksberg C, Denmark. 'Center for Synthetic Biology "bioSYNergy", Department of Plant and Environmental Sciences, University of Copenhagen, Thorvaldsensvej 40, DK-1871 Frederiksberg C, Denmark. ${ }^{3}$ Department of Drug Design and Pharmacology, Faculty of Health and Medical Sciences, University of Copenhagen, DK-2100 Copenhagen, Denmark.

Received: 19 November 2019 Accepted: 17 February 2020

Published online: 28 February 2020

\section{References}

1. Chinnock RJ. Eremophila and allied genera. A monograph of the Myoporaceae. Australia: Rosenberg Publishing; 2007.

2. Barr A. Traditional bush medicines: an aboriginal pharmacopoeia. Richmond: Greenhouse Publications; 1988.

3. Lassak E, McCarthy T. Australian medicinal plants: a complete guide to identification and usage. 2nd Editio. Chatswood: Reed New Holland; 2011.

4. Hansen V, Horsfall J. Noongar bush medicine plants: medicinal plants of the south-west of Western Australia. Crawley: UWA Publishing; 2016.

5. Isaacs J. Bush food: aboriginal food and herbal medicine. McMahons Point: Weldons; 1987.

6. Semple SJ, Reynolds GD, O'Leary MC, Flower RLP. Screening of Australian medicinal plants for antiviral activity. J Ethnopharmacol. 1998;60:163-72.

7. Palombo EA, Semple SJ. Antibacterial activity of traditional Australian medicinal plants. J Ethnopharmacol. 2001;77:151-7

8. Palombo EA, Semple SJ. Antibacterial activity of Australian plant extracts against methicillin-resistant Staphylococcus aureus (MRSA) and vancomycinresistant enterococci (VRE). J Basic Microbiol. 2002:42:444-8.

9. Ndi CP, Semple SJ, Griesser HJ, Barton MD. Antimicrobial activity of some Australian plant species from the genus Eremophila. J Basic Microbiol. 2007:47:158-64.

10. Mijajlovic S, Smith J, Watson K, Parsons P, Jones GL. Traditional Australian medicinal plants: screening for activity against human cancer cell lines. J Aust Tradit-Med Soc. 2006;12:129-32.

11. Rogers KL, Fong WF, Redburn J, Griffiths LR. Fluorescence detection of plant extracts that affect neuronal voltage-gated Ca2+ channels. Eur J Pharm Sci. 2002;15:321-30.

12. Kumar R, Duffy S, Avery VM, Carroll AR, Davis RA. Microthecaline a, a quinoline serrulatane alkaloid from the roots of the Australian desert plant Eremophila microtheca. J Nat Prod. 2018;81:1079-83. 
13. Liu Q, Harrington D, Kohen JL, Vemulpad S, Jamie JF. Bactericidal and cyclooxygenase inhibitory diterpenes from Eremophila sturtii. Phytochemistry. 2006;67:1256-61.

14. Ndi CP, Semple SJ, Griesser HJ, Pyke SM, Barton MD. Antimicrobial compounds from the Australian desert plant Eremophila neglecta. J Nat Prod. 2007;70:1439-43.

15. Barnes EC, Kavanagh AM, Ramu S, Blaskovich M, Cooper M, Davis R. Antibacterial serrulatane diterpenes from the Australian native plant Eremophila microtheca. Phytochemistry. 2013;93:162-9.

16. Mon HH, Christo SN, Ndi CP, Jasieniak M, Rickard H, Hayball JD, et al. Serrulatane diterpenoid from Eremophila neglecta exhibits bacterial biofilm dispersion and inhibits release of pro-inflammatory cytokines from activated macrophages. J Nat Prod. 2015;78:3031-40.

17. Algreiby AA, Hammer KA, Durmic Z, Vercoe P, Flematti GR. Antibacterial compounds from the Australian native plant Eremophila glabra. Fitoterapia. 2018;126:45-52

18. Tahtah Y, Wubshet SG, Kongstad KT, Heskes AM, Pateraki I, Møller BL, et al. High-resolution PTP1B inhibition profiling combined with high-performance liquid chromatography-high-resolution mass spectrometry-solid-phase extraction-nuclear magnetic resonance spectroscopy: proof-of-concept and antidiabetic constituents in crude extract of Eremophila lucida. Fitoterapia. 2016;110:52-8

19. Wubshet SG, Tahtah Y, Heskes AM, Kongstad KT, Pateraki I, Hamberger B, et al. Identification of PTP1B and a-glucosidase inhibitory serrulatanes from Eremophila spp. by combined use of dual high-resolution PTP1B and aglucosidase inhibition profiling and HPLC-HRMS-SPE-NMR. J Nat Prod. 2016; 79:1063-72.

20. Molina-Salinas GM, Rivas-Galindo VM, Said-Fernández S, Lankin DC, Muñoz MA, Joseph-Nathan P, et al. Stereochemical analysis of leubethanol, an antiTB active serrulatane, from Leucophyllum frutescens. J Nat Prod. 2011;74: 1199-216.

21. Berrue F, Kerr RG. Diterpenes from gorgonian corals. Nat Prod Rep. 2009;26: $681-710$.

22. Singab AN, Youssef FS, Ashour ML, Wink M. The genus Eremophila (Scrophulariaceae): an ethnobotanical, biological and phytochemical review. J Pharm Pharmacol. 2013:65:1239-79.

23. Peters RJ. Two rings in them all: the labdane-related diterpenoids. Nat Prod Rep. 2010;27:1521-30.

24. Bohlmann J, Meyer-Gauen G, Croteau R. Plant terpenoid synthases: molecular biology and phylogenetic analysis. Proc Natl Acad Sci. 1998;95: 4126-33.

25. Chen F, Tholl D, Bohlmann J, Pichersky E. The family of terpene synthases in plants: a mid-size family of genes for specialized metabolism that is highly diversified throughout the kingdom. Plant J. 2011;66:212-29.

26. Zerbe P, Bohlmann J. Plant diterpene synthases: exploring modularity and metabolic diversity for bioengineering. Trends Biotechnol. 2015;33:419-28.

27. Zerbe P, Hamberger B, Yuen MMS, Chiang A, Sandhu HK, Madilao LL, et al Gene discovery of modular diterpene metabolism in nonmodel systems. Plant Physiol. 2013;162:1073-91.

28. Falara V, Alba JM, Kant MR, Schuurink RC, Pichersky E. Geranyllinalool synthases in Solanaceae and other angiosperms constitute an ancient branch of diterpene synthases that are involved in the synthesis of defensive compounds. Plant Physiol. 2014;166:428-41.

29. Kirby J, Nishimoto M, Park JG, Withers ST, Nowroozi F, Behrendt D, et al. Cloning of casbene and neocembrene synthases from Euphorbiaceae plants and expression in Saccharomyces cerevisiae. Phytochemistry. 2010;71: 1466-73.

30. Wang Q, Jia M, Huh J-H, Muchlinski A, Peters RJ, Tholl D. Identification of a dolabellane type diterpene synthase and other root-expressed diterpene synthases in Arabidopsis. Front Plant Sci. 2016;7:1761.

31. Xiong W, Wu P, Jia Y, Wei X, Xu L, Yang Y, et al. Genome-wide analysis of the terpene synthase gene family in physic nut (Jatropha curcas L.) and functional identification of six terpene synthases. Tree Genet Genomes. 2016;12:97.

32. Matsuba Y, Nguyen TTH, Wiegert $K$, Falara V, Gonzales-Vigil E, Leong B, et al. Evolution of a complex locus for terpene biosynthesis in Solanum. Plant Cell. 2013;25:2022-36.

33. Vaughan MM, Wang Q, Webster FX, Kiemle D, Hong YJ, Tantillo DJ, et al. Formation of the unusual semivolatile diterpene rhizathalene by the Arabidopsis class I terpene synthase TPS08 in the root stele is involved in defense against belowground herbivory. Plant Cell. 2013;25:1108-25.
34. Zi J, Matsuba Y, Hong YJ, Jackson AJ, Tantillo DJ, Pichersky E, et al. Biosynthesis of lycosantalonol, a cis-prenyl derived diterpenoid. J Am Chem Soc. 2014;136:16951-3.

35. Johnson SR, Bhat WW, Sadre R, Miller GP, Sky A, Hamberger B. Promiscuous terpene synthases from Prunella vulgaris highlight the importance of substrate and compartment switching in terpene synthase evolution. New Phytol. 2019;223:323-35.

36. Christianson DW. Structural and chemical biology of terpenoid cyclases. Chem Rev. 2017;117:11570-648.

37. Nagel R, Bernholz C, Vranov E, Bergau N, Ludwig S, Wessjohann L, et al. Arabidopsis thaliana isoprenyl diphosphate synthases produce the C25 intermediate geranylfarnesyl diphosphate. Plant J. 2015;84:847-59.

38. Liu Y, Luo S-H, Schmidt A, Wang G-D, Sun G-L, Grant M, et al. A geranylfarnesyl diphosphate synthase provides the precursor for sesterterpenoid (C25) formation in the glandular trichomes of the mint species Leucosceptrum canum. Plant Cell. 2016;28:804-22

39. Sallaud C, Rontein D, Onillon S, Jabès F, Duffé P, Giacalone C, et al. A novel pathway for sesquiterpene biosynthesis from Z,Z-farnesyl pyrophosphate in the wild tomato Solanum habrochaites. Plant Cell. 2009;21:301-17.

40. Schilmiller AL, Schauvinhold I, Larson M, Xu R, Charbonneau AL, Schmidt A, et al. Monoterpenes in the glandular trichomes of tomato are synthesized from a neryl diphosphate precursor rather than geranyl diphosphate. Proc Natl Acad Sci. 2009;106:10865-70.

41. Falara V, Akhtar TA, Nguyen TTH, Spyropoulou EA, Bleeker PM, Schauvinhold I, et al. The tomato terpene synthase gene family. Plant Physiol. 2011;157: 770-89.

42. Bleeker PM, Mirabella R, Diergaarde PJ, VanDoorn A, Tissier A, Kant MR, et al. Improved herbivore resistance in cultivated tomato with the sesquiterpene biosynthetic pathway from a wild relative. Proc Natl Acad Sci. 2012;109: 20124-9.

43. Gonzales-vigil E, Hufnagel DE, Kim J, Last RL, Barry CS. Evolution of TPS20related terpene synthases influences chemical diversity in the glandular trichomes of the wild tomato relative Solanum habrochaites. Plant J. 2012; 71:921-35.

44. Matsuba Y, Zi J, Jones AD, Peters RJ, Pichersky E. Biosynthesis of the diterpenoid lycosantalonol via nerylneryl diphosphate in Solanum lycopersicum. PLoS One. 2015;10:e0119302.

45. Grabińska KA, Park EJ, Sessa WC. cis-Prenyltransferase: new insights into protein glycosylation, rubber synthesis, and human diseases. J Biol Chem. 2016;291:18582-90.

46. Asawatreratanakul K, Zhang Y, Wititsuwannakul D, Wititsuwannakul R, Takahashi S, Rattanapittayaporn A, et al. Molecular cloning, expression and characterization of cDNA encoding cis-prenyltransferases from Hevea brasiliensis. A key factor participating in natural rubber biosynthesis. Eur J Biochem. 2003:4680:4671-80.

47. Schmidt T, Hillebrand A, Wurbs D. Molecular cloning and characterization of rubber biosynthetic genes from Taraxacum koksaghyz. Plant Mol Biol Report. 2010;28:277-84.

48. Qu Y, Chakrabarty R, Tran HT, Kwon EJG, Kwon M, Nguyen TD, et al. A lettuce (Lactuca sativa) homolog of human nogo-B receptor interacts with cis-prenyltransferase and is necessary for natural rubber biosynthesis. J Biol Chem. 2015;290:1898-914

49. Akhtar T, Przemyslaw S, Siekierska H, Kania M, Gelder K, Rea KA, et al. Polyprenols are synthesized by a plastidial cis-prenyltransferase and influence photosynthetic performance. Plant Cell. 2017;29:1709-25.

50. Akhtar TA, Matsuba Y, Schauvinhold I, Yu G, Lees HA, Klein SE, et al. The tomato cis-prenyltransferase gene family. Plant J. 2013;73:640-52.

51. Kracht ON, Ammann A-C, Stockmann J, Wibberg D, Kalinowski J, Piotrowski $M$, et al. Transcriptome profiling of the Australian arid-land plant Eremophila serrulata (a.DC.) Druce (Scrophulariaceae) for the identification of monoterpene synthases. Phytochemistry. 2017;136:15-22.

52. Hutton PG, Durmic Z, Ghisalberti EL, Flematti GR, Duncan RM, Carson CF, et al. Inhibition of ruminal bacteria involved in lactic acid metabolism by extracts from Australian plants. Anim Feed Sci Technol. 2012:176:170-7.

53. Croft KD, Ghisalberti EL, Jefferies PR, Proudfoot GM. The chemistry of Eremophila spp. XVI new serrulatanes from Eremophila spp. Aust J Chem. 1981;34:1951-7

54. Forster PG, Ghisalberti EL, Jefferies PR, Poletti VM, Whiteside NJ. Serrulatane diterpenes from Eremophila spp. Phytochemistry. 1986;25:1377-83.

55. Tissier A. Plant secretory structures: more than just reaction bags. Curr Opin Biotechnol. 2018;49:73-9. 
56. Kumar S, Stecher G, Tamura K. MEGA7: molecular evolutionary genetics analysis version 7.0 for bigger datasets. Mol Biol Evol. 2016;33:1870-4.

57. Almagro Armenteros JJ, Sønderby CK, Sønderby SK, Nielsen H, Winther O. DeepLoc: prediction of protein subcellular localization using deep learning Bioinformatics. 2017;33:3387-95.

58. Brückner K, Tissier A. High-level diterpene production by transient expression in Nicotiana benthamiana. Plant Methods. 2013;9:46-56.

59. Bach SS, Bassard J-É, Andersen-Ranberg J, Møldrup ME, Simonsen HT, Hamberger B. High-throughput testing of terpenoid biosynthesis candidate genes using transient expression in Nicotiana benthamiana. In: RodríguezConcepción M, editor. Plant Isoprenoids. Methods in molecular biology (Methods and Protocols). New York: Humana Press; 2014. p. 285-99.

60. Andersen-Ranberg J, Kongstad KT, Nielsen MT, Jensen NB, Pateraki I, Bach SS, et al. Expanding the landscape of diterpene structural diversity through stereochemically controlled combinatorial biosynthesis. Angew Chem. 2016;128:2182-6.

61. Epping J, van Deenen N, Niephaus E, Stolze A, Fricke J, Huber C, et al. A rubber transferase activator is necessary for natural rubber biosynthesis in dandelion. Nature Plants. 2015;1:15048.

62. Brasher MI, Surmacz L, Leong B, Pitcher J, Swiezewska E, Pichersky E, et al. A two-component enzyme complex is required for dolichol biosynthesis in tomato. Plant J. 2015;82:903-14.

63. Gelder KV, Rea KA, Virta LKA, Whitnell KL, Osborn M, Vatta M, et al. Mediumchain polyprenols influence chloroplast membrane dynamics in Solanum Iycopersicum. Plant Cell Physiol. 2018;59:2350-65.

64. Demissie ZA, Erland LAE, Rheault MR, Mahmoud SS. The biosynthetic origin of irregular monoterpenes in Lavandula: isolation and biochemical characterization of a novel cis-prenyl diphosphate synthase gene, lavandulyl diphosphate synthase. J Biol Chem. 2013;288:6333-41.

65. Dell B. Distribution and function of resins and glandular hairs in Western Australian plants. J R Soc West Aust. 1977;59:119-23.

66. Ghisalberti EL, Jefferies PR, Knox JR, Sheppard PN. The chemistry of Eremophila SP-VII: an epoxycembradienol from Eremophila georgei. Tetrahedron. 1977;33:3301-3.

67. Ghisalberti EL. The chemistry of unusual terpenoids from the genus Eremophila. In: Atta-ur-Rahman, editor. Studies in Natural Products Chemistry: Elsevier; 1995. p. 225-87.

68. Zager JJ, Lange BM. Assessing flux distribution associated with metabolic specialization of glandular trichomes. Trends Plant Sci. 2018;23:638-47.

69. Lange BM, Turner GW. Terpenoid biosynthesis in trichomes - current status and future opportunities. Plant Biotechnol J. 2013;11:2-22.

70. Tissier A. Harnessing plant trichome biochemistry for the production of useful compounds. In: Kermode AR, editor. Molecular pharming: applications, challenges, and emerging areas. Hoboken: Wiley; 2018. p. 353-82

71. Liu Y, Jing S-X, Luo S-H, Li S-H. Non-volatile natural products in plant glandular trichomes: chemistry, biological activities and biosynthesis. Nat Prod Rep. 2019:36:626-65.

72. Zi J, Mafu S, Peters RJ. To gibberellins and beyond! Surveying the evolution of (di) terpenoid metabolism. Annu Rev Plant Biol. 2014;65:259-86.

73. Mau CJD, West CA. Cloning of casbene synthase CDNA: evidence for conserved structural features among terpenoid cyclases in plants. Proc Natl Acad Sci. 1994;91:8497-501.

74. King AJ, Brown GD, Gilday AD, Larson TR, Graham IA. Production of bioactive diterpenoids in the Euphorbiaceae depends on evolutionarily conserved gene clusters. Plant Cell. 2014:26:3286-98.

75. Luo D, Callari R, Hamberger B, Wubshet SG, Nielsen MT, Andersen-Ranberg J, et al. Oxidation and cyclization of casbene in the biosynthesis of Euphorbia factors from mature seeds of Euphorbia lathyris L. Proc Natl Acad Sci. 2016;113:E5082-9.

76. Hansen NL, Heskes AM, Hamberger B, Olsen CE, Hallström BM, AndersenRanberg J, et al. The terpene synthase gene family in Tripterygium wilfordii harbors a labdane-type diterpene synthase among the monoterpene synthase TPS-b subfamily. Plant J. 2017;89:429-41.

77. Inabuy FS, Fischedick JT, Lange I, Hartmann M, Srividya N, Parrish AN, et al. Biosynthesis of diterpenoids in Tripterygium adventitious root cultures. Plant Physiol. 2017;175:92-103.

78. Nagegowda DA, Gutensohn M, Wilkerson CG, Dudareva N, Lafayette W, Consortium MP, et al. Two nearly identical terpene synthases catalyze the formation of nerolidol and linalool in snapdragon flowers. Plant J. 2008;55:224-39.

79. Huang M, Abel C, Sohrabi R, Petri J, Haupt I, Cosimano J, et al. Variation of herbivore-induced volatile terpenes among Arabidopsis ecotypes depends on allelic differences and subcellular targeting of two terpene synthases, TPS02 and TPS03. Plant Physiol. 2010;153:1293-310.

80. Jones CG, Moniodis J, Zulak KG, Scaffidi A, Plummer JA, Ghisalberti EL, et al. Sandalwood fragrance biosynthesis involves sesquiterpene synthases of both the terpene synthase (TPS)-a and TPS-b subfamilies, including santalene synthases. J Biol Chem. 2011;286:17445-54.

81. Jia M, Mishra SK, Tufts S, Jernigan RL, Peters RJ. Combinatorial biosynthesis and the basis for substrate promiscuity in class I diterpene synthases. Metab Eng. 2019;55:44-58.

82. Pazouki L, Niinemets Ü. Multi-substrate terpene synthases: their occurrence and physiological significance. Front Plant Sci. 2016;7:1-16.

83. Jia M, Potter KC, Peters RJ. Extreme promiscuity of a bacterial and a plant diterpene synthase enables combinatorial biosynthesis. Metab Eng. 2016;37: 24-34.

84. Jia M, Peters RJ. cis or trans with class II diterpene cyclases. Org Biomol Chem. 2017;15:3158-60.

85. Greenhagen BT, Maille PEO, Noel JP, Chappell J. Identifying and manipulating structural determinates linking catalytic specificities in terpene synthases. Proc Natl Acad Sci. 2006;103:9826-31.

86. Schrepfer P, Buettner A, Goerner C, Hertel M, van Rijn J, Wallrapp F, et al. Identification of amino acid networks governing catalysis in the closed complex of class I terpene synthases. Proc Natl Acad Sci. 2016;113:E958-67.

87. Hansen NL, Nissen JN, Hamberger B. Two residues determine the product profile of the class II diterpene synthases TPS14 and TPS21 of Tripterygium wilfordii. Phytochemistry. 2017;138:52-6.

88. Noel P, Dellas N, Faraldos J, Zhao M, Hess B, Smentek L, et al. Structural elucidation of cisoid and transoid cyclization pathways of a sesquiterpene synthase using 2-fluorofarnesyl diphosphates. ACS Chem Biol. 2010;5:377-92.

89. Faraldos JA, O'Maille PE, Dellas N, Noel JP, Coates RM. Bisabolyl-derived sesquiterpenes from tobacco 5-epi-aristolochene synthase-catalyzed cyclization of (2Z,6E)-farnesyl diphosphate. J Am Chem Soc. 2010;132:4281-9.

90. Garms S, Köllner TG, Boland W. A multiproduct terpene synthase from Medicago truncatula generates cadalane sesquiterpenes via two different mechanisms. J Organomet Chem. 2010;75:5590-600.

91. Faraldos JA, Miller DJ, Li A, Allemann RK. A 1,6-ring closure mechanism for (+)- $\delta$-cadinene synthase? J Am Chem Soc. 2012;134:5900-8.

92. Forster PG, Ghisalberti EL, Jefferies PR. A new class of monocyclic diterpene from Eremophila foliosissima Kraenzlin. (Myoporaceae). Tetrahedron. 1987:43: 2999-3007.

93. Forster PG, Ghisalberti EL, Jefferies PR. A new cedrene isoprenologue from the resin of Eremophila georgei. J Nat Prod. 1993;56:147-52.

94. Aminimoghadamfarouj N, Nematollahi A. Structure elucidation and botanical characterization of diterpenes from a specific type of bee glue. Molecules. 2017;22:1185.

95. Matasci N, Hung L, Yan Z, Carpenter EJ, Wickett NJ, Mirarab S, et al. Data access for the 1000 plants (1KP) project. GigaScience. 2014;3:17.

96. Tank DC, Beardsley PM, Kelchner SA, Olmstead RG. Review of the systematics of Scrophulariaceae s.l. and their current disposition. Aust Syst Bot. 2006;19:289-307.

97. Dell B, McComb AJ. Glandular hair formation and resin secretion in Eremophila fraseri F. Meull (Myoporaceae). Protoplasma. 1977;92:71-86.

98. Scheler U, Rothe K, Manzano D, Bozic D, Papaefthimiou D, Balcke GU, et al. Engineering the biosynthesis of carnosic acid and carnosol in yeast. Nat Commun. 2016;7:12942.

99. Ignea C, Athanasakoglou A, loannou E, Georgantea P, Trikka FA, Loupassaki $S$, et al. Carnosic acid biosynthesis elucidated by a synthetic biology platform. Proc Natl Acad Sci. 2016;113:3681-6.

100. Haas BJ, Papanicolaou A, Yassour M, Grabherr M, Blood PD, Bowden J, et al. De novo transcript sequence reconstruction from RNA-seq using the trinity platform for reference generation and analysis. Nat Protoc. 2013:8:1494-512.

101. Bray NL, Pimentel H, Melsted P, Pachter L. Near-optimal probabilistic RNAseq quantification. Nat Biotechnol. 2016;34:525-8.

102. Heskes AM, Sundram TCM, Boughton BA, Jensen NB, Hansen NL, Crocoll C, et al. Biosynthesis of bioactive diterpenoids in the medicinal plant Vitex agnus-castus. Plant J. 2018:93:943-58.

103. Nour-Eldin HH, Geu-Flores F, Halkier BA. USER cloning and USER fusion: The ideal cloning techniques for small and big laboratories. In: Fett-Neto A, editor. Plant Secondary Metabolism Engineering. Methods in Molecular Biology (Methods and Protocols). Totowa; 2010. https://doi.org/10.1007/9781-60761-723-5. 
104. Baulcombe DC, Molnár A. Crystal structure of p19 - a universal suppressor of RNA silencing. Trends Biochem Sci. 2004;29:279-81.

105. Morrone D, Lowry L, Determan MK, Hershey DM, Xu M, Peters RJ. Increasing diterpene yield with a modular metabolic engineering system in $E$. coli: comparison of MEV and MEP isoprenoid precursor pathway engineering. Appl Microbiol Biotechnol. 2010;85:1893-906.

106. Cyr A, Wilderman PR, Determan M, Peters RJ. A modular approach for facile biosynthesis of labdane-related diterpenes. J Am Chem Soc. 2007;129:6684-5.

\section{Publisher's Note}

Springer Nature remains neutral with regard to jurisdictional claims in published maps and institutional affiliations.

Ready to submit your research? Choose BMC and benefit from:

- fast, convenient online submission

- thorough peer review by experienced researchers in your field

- rapid publication on acceptance

- support for research data, including large and complex data types

- gold Open Access which fosters wider collaboration and increased citations

- maximum visibility for your research: over $100 \mathrm{M}$ website views per year

At $\mathrm{BMC}$, research is always in progress.

Learn more biomedcentral.com/submissions 